\title{
Facilitation of recall with intralist cues: Time-dependent characteristics of cued recall
}

\author{
JOELLEN T. HARTLEY \\ University of California, Irvine, California 92664
}

\begin{abstract}
List items were given as retrieval cues in a free-recall experiment which factorially combined the presence or absence of cues with the amount of time allowed for use of each cue (10 sec or $30 \mathrm{sec})$. A categorizable list of 75 randomly presented words was learned, and $48 \mathrm{~h}$ later a free-recall test trial was given, followed by a final memory search task. During the final task, cued subjects received words from categories that had not been recalled during the free-recall test. With both time intervals, cued subjects recalled more words than noncued subjects, indicating that random presentation of categorized words does not necessarily preclude the observation of a cueing effect with list items, as has been reported previously. The composition of recall, whether from previously recalled or nonrecalled categories, varied as a function of time for both groups. The results were interpreted in terms of retrieval strategies employed by cued and noncued subjects and the effect of time on these strategies.
\end{abstract}

Acceptance of the theoretical distinction between storage and retrieval of information in memory focused a good deal of attention on the categorization of various memory phenomena as reflecting either storage processes or retrieval processes. In free recall, the phenomenon of output organization observed as either category clustering of taxonomically related words (Bousfield, 1953 ) or subjective organization of unrelated words (Tulving, 1962) was of particular interest. For a period of time, the argument about whether this phenomenon reflected storage processes (a trace-dependent hypothesis) or retrieval processes (a trace-independent hypothesis) centered upon the effectiveness of list items themselves as retrieval cues for additional list items. The logic was simply that, if list items are stored together (dependent traces), then providing one item (an intralist cue) should guarantee access to others (Slamecka, 1968).

In a series of experiments wilich provided the impetus for research on intralist retrieval cues, Slamecka (1968) found no evidence of increased recall when cues were given in a variety of conditions. With lists of unrelated words, related words, and categorized words, providing intralist cues did not increase recall when cued and noncued conditions were compared. These results were interpreted by Slamecka as indicating that a tracedependency theory of item storage was untenable.

This research was supported by National Institute of Mental Health Predoctoral Fellowship 1 F01 MH45763-03. The author expresses appreciation to Isabel Birnbaum for critical comments during all phases of the research and to Alan Hartley and Elizabeth Parker for comments and suggestions on earlier versions of this manuscript. Author's current address: Department of Psychology, Florida International University, Miami, Florida 33199 .
The original work on intralist cues (Slamecka, 1968) has been criticized on the basis of certain procedural difficulties (e.g., Wood, 1969), and a number of investigators have since demonstrated that intralist cues can be effective in both single-trial and multitrial free recall, but only under limited conditions. When related words or categorized words were blocked together during presentation, intralist cues were shown to facilitate recall; when the same list was presented in a random order, intralist cues failed to facilitate recall (Allen, 1969; Lewis, 1971; Luek, McLaughlin, \& Cicala, 1971; Wood, 1969). Hudson and Austin (1970) reported a study in which explicit information about the categorical structure of the list was given (along with category names) prior to random presentation of category instances. Facilitation by intralist cues was observed in only one of their experiments, and the effect appeared to be restricted to the first recall trial only. In general, with related or categorized words, intralist cues have been effective only when presentation or instructional procedures were employed which made the inherent list structure highly salient at the outset of learning.

While failure to find a positive cueing effect with intralist cues could be viewed as evidence against a trace-dependency hypothesis, the successful demonstration of an effect of intralist cues does not resolve the trace-dependency, trace-independency controversy. If a list item does act as a cue for the retrieval of another list item, this fact does not in itself imply dependency of the two traces in storage. Rather, the one item may activate a retrieval strategy which then operates on the second item which could be stored independently. The more recent theoretical interest in cueing is related to the notions of encoding specificity as developed by Tulving and Thomson (1973). According to this posi- 
tion, any item given as a cue (including an intralist cue) will facilitate recall only if that item was encoded with the list items at the time of input. Failure to find a positive effect of intralist cues with random presentation of categorized words, when the effect is relatively reliable with blocked presentation, may indicate that encoding strategies in blocked and random presentation are markedly different. On the other hand, the inability to demonstrate a positive cueing effect with random presentation could reflect a methodological inadequacy rather than processing differences.

In all studies which have shown facilitation with intralist cues, the primary difference between cued and noncued groups was that the cued group recalled more categories while the number of words recalled within each category was the same (Hudson \& Austin, 1970; Slamecka, 1972; Wood, 1969). It has been observed, however, that category recall is superior following random presentation when compared to blocked presentation (Borges \& Mandler, 1972; Hartley, 1974; Lewis, 1974). Since a demonstration of cueing requires a difference between cued and noncued subjects treated otherwise identically, it is possible that providing intralist cues to random presentation groups has been ineffective largely because these groups had fewer inaccessible categories than corresponding blocked presentation groups. A similar analysis has been suggested by Lewis (1974). In order to observe a cueing effect, therefore, a discrepancy must exist between the number of categories potentially available and the number recalled unaided. If these conditions can be met with random presentation, then intralist cues should facilitate recall.

Birnbaum and Eichner (1971) used a technique designed to maximize the probability of observing a cueing effect. Intralist cues were words from categories which had not been recalled during the preceding free-recall period; thus, the cues allowed access to potentially available but nonrecalled categories. While a positive cueing effect was observed, a chance difference between the cued and noncued groups in number of categories available at the time of cueing (more were available for the cued group) precluded an unambiguous conclusion. An unpublished analysis, however, revealed interesting differences in the composition of recall for cued and noncued subjects. Noncued subjects produced new items primarily from categories which had been previously recalled during the initial free-recall period, while cued subjects retrieved words associated with the cues almost exclusively, and failed to produce additional words from previously recalled categories. These data indicate that cued subjects may have been unable to abandon a particular search strategy once it had been initiated, suggesting that retrieval cues may actually have a negative effect on memory search after an initial facilitation. Alternatively, the differences may simply indicate that a thorough search of memory was prevented by the time restrictions imposed during the cueing period (10 sec/cue). If more time is allowed, cued subjects may be as successful as noncued subjects in the retrieval of items from previously recalled categories.

The present study was undertaken to resolve the ambiguities of the cueing data from Birnbaum and Eichner (1971) and to provide support for the conclusion that intralist cues will facilitate recall following random presentation of categorized words. A second purpose was to determine whether time constraints account for the observed differences in composition of recall for cued and noncued subjects.

\section{METHOD}

\section{Design}

A 2 by 2 factorial between-subjects design was used with cueing (cued vs. noncued) and time (10 sec vs. $30 \mathrm{sec}$ ) per cue as variables. The experiment was conducted in two sessions. During the first session, all subjects received four standard freerecall learning cycles of alternating study and test trials followed by one final study trial. The second session was conducted $48( \pm 2) h$ later and consisted of an unaided free-recall test (Phase 1) followed by a period of either cued or noncued recall, with either $10 \mathrm{sec}$ or $30 \mathrm{sec}$ allowed per cue of the task (Phase 2).

\section{Materials}

A single list of 75 words was used, composed of 3 words from each of 25 categories chosen from the category norms of Battig and Montague (1969). The list was identical to that used by Birnbaum and Eichner (1971). The items varied in response frequency from 100 to 351 in the norms. Items which could be perceived as belonging to more than one category on the list were excluded. Random orders of the words were constructed with the restriction that no two items from the same category occurred adjacently. One item from each category was randomly selected and designated as a cue word for Phase 2 of the delayed recall task.

\section{Procedure}

Upon entering the laboratory for the first session, all subjects received standard free-recall learning instructions. The list was presented by audio tape at a 1.5 -sec rate. Four different random orders of the list were used equally often as the first study order. Study and test trials were each $112 \mathrm{sec}$, with an 8-sec intertrial interval. Recall was oral, and the experimenter wrote the words down as they were called out. A tape recording was made during each test trial so that the accuracy of the written record could be verified at a later time. Immediately after the final study trial, all subjects received instructions for a nonverbal filler task which continued for $2 \mathrm{~min}$. This task was included to reproduce as closely as possible the procedures used by Birnbaum and Eichner (1971). Participants were then dismissed with instructions to return in 2 days for the second part of the experiment. No information about the second session was given at this time.

Upon return to the laboratory, the subjects were given instructions for a free-recall test trial (Phase 1) and allowed to engage in oral recall for $172 \mathrm{sec}, 60 \mathrm{sec}$ longer than previous test trials. The additional time was allowed to encourage production of as many words as possible during the unaided portion of the final test. Phase 2 instructions were read $90 \mathrm{sec}$ after Phase 1 ended, and written recall began immediately after the instructions were read. A total of 10 pages were handed to each subject, one at a time, with $5 \mathrm{sec}$ allowed between pages for passing the sheets. For the cued conditions, one word was printed at the top of each page. For the noncued conditions, the pages were blank. Depending upon the experimental group, 
subjects were allowed 10 or $30 \mathrm{sec} /$ page. For each group, the subjects had been fully informed of the nature of the cueing condition and the amount of time allowed per page. All subjects were instructed to attempt to recall those words which they had not recalled during Phase 1 . They were told that if they were uncertain about a word, they should write it down. Whenever possible, a cue word was given from a category which had not been accessed during Phase 1; therefore, each cued subject received a different set of 10 items. When fewer than 10 categories had been forgotten, additional cues were selected at random from categories already recalled. Likewise, when more than 10 categories had been forgotten, a random process was used to select the cues.

\section{Subjects}

The subjects were 64 students at the University of California, Irvine, who chose to participate in the experiment to fulfill a course requirement. A total of 16 blocks were constructed from the four experimental groups. The order of testing groups within blocks was randomly determined. The subjects were tested individually and were assigned to groups in order of appearance in the laboratory.

\section{RESULTS}

All analyses reported below were two-factor analyses including Cueing (cued vs. noncued) and Time (10 vs. 30 sec) as between-subjects variables. An analysis of variance on the acquisition data of the first session showed no differences due to either experimental variable (ps $>.25$ ), indicating that learning differences were not a factor of interest.

\section{Phase 1 Recall}

Table 1 shows the number of items correctly recalled for all groups during Phase 1 and Phase 2 of the delayed recall test. Analysis of variance on number of items recalled during Phase 1 showed no differences for Cueing or Time, all Fs $<1.0$ (for all effects, $\mathrm{MS}_{\mathrm{e}}=146.3$ ). The second measure of interest was the number of categories recalled by cued and noncued groups. An analysis of variance indicated no significant differences for Time, $\mathrm{F}(1,60)<1.0$, or Cueing, $\mathrm{F}(1,60)=1.78, \mathrm{p}<.25$ (for all effects, $\mathrm{MS}_{\mathrm{e}}=8.9$ ). For the cued groups, the mean number of categories recalled was 17.6 during Phase 1 and, for the noncued groups, the mean was 18.6. It can be concluded that all groups were equivalent prior to Phase 2 recall.

\section{Phase 2 Scoring}

The new words recalled by each subject were partitioned into two subsets: those from categories that had been recalled during Phase 1 ( $R C$ items) and those from

Table 1

Number of Items Recalled Correctly on 48-H Retention Test

\begin{tabular}{lccc}
\hline Group & Time (sec) & Phase 1 & Phase 2 \\
\hline \multirow{2}{*}{ Cued } & 10 & 41.12 & 8.94 \\
& 30 & 43.75 & 10.94 \\
\multirow{2}{*}{ Noncued } & 10 & 43.62 & 3.62 \\
& 30 & 42.37 & 7.38 \\
\hline
\end{tabular}

Table 2

Mean Proportion of Items Recalled from RC and NRC Pools During Phase 2 of Retention Test

\begin{tabular}{lccc}
\hline & Time & \multicolumn{2}{c}{ Item Type } \\
Group & $(\mathrm{sec})$ & RC & NRC \\
\hline \multirow{2}{*}{ Cued } & 10 & .02 & .63 \\
& 30 & .28 & .71 \\
\multirow{2}{*}{ Noncued } & 10 & .22 & .10 \\
& 30 & .25 & .42 \\
\hline
\end{tabular}

categories that had not been recalled during Phase 1 (NRC items). In addition, the 25 list words which were potential Phase 2 cues were designated as noncritical items, and recall of any of these words during Phase 2 was not included in the score for either cued or noncued subjects. For each subject, the pool of possible RC items was tabulated. These were all nonrecalled items belonging to categories represented in Phase 1 by one or two words (minus noncritical items which fell into this class). Thus, it was possible that there could be one remaining item in a category; but if that item was a potential cue word, it was not tabulated as a member of the RC-item pool. The pool of possible NRC items consisted of all words from categories which had not been represented by one word during Phase 1 (minus the noncritical items). The same scoring procedure was used for cued and noncued Phase 2 protocols.

\section{Phase 2 Recall}

It is apparent from inspection of Table 1 that intralist cues facilitated retrieval during Phase 2 . An analysis of variance revealed two significant effects: Cueing, $F(1,60)$ $=23.07, p<.01$, and Time, $F(1,60)=9.68, p<.01$ (for all effects, $M S_{e}=13.6$ ). The interaction of Time by Cueing was not significant, $F<1.0$. Cued subjects recalled an average of 9.94 new words, while noncued subjects recalled 5.50 new words. Both cued and noncued subjects recalled more new words when $30 \mathrm{sec}$ were allowed per page.

\section{Item-Type Analysis}

For each subject, the RC items and NRC items recalled in Phase 2 were expressed as a proportion of the total possible for each of the classifications. Since the number of categories that had not been recalled during Phase 1 was the same for all groups, the pool of possible NRC items was equivalent for all groups. An analysis of variance also indicated that the pool of possible RC items was the same for all groups prior to Phase 2. Table 2 shows the mean proportion of each type of item recalled during Phase 2.

Turning first to NRC-item recall, it is apparent that cued subjects were superior to noncued subjects for this type of item. Following an arc sine transformation (recommended by Kirk, 1968, p. 66), the data were submitted for analysis of variance. Both the main effects of Time and Cueing were significant, as well as the interaction of Time by Cueing: Time, $F(1,60)=9.27$, 
$\mathrm{p}<.01$; Cueing, $\mathrm{F}(1,60)=37.59, \mathrm{p}<.01$; and Time by Cueing, $F(1,60)=4.49, \mathrm{p}<.05$ (for all effects, $\mathrm{MS}_{\mathrm{e}}=$ .4). A test for simple main effects (Kirk, 1968, pp. 179-182) was performed to determine the nature of the interaction. Four such tests were done, and the overall $\alpha$ of .05 was divided evenly among the simple maineffect tests. The results revealed that the effect of Cueing was significant at both levels of the time variable and that the effect of Time was significant only for the noncued level of the cueing variable. List item cues from nonrecalled categories were highly effective in providing access to forgotten units, as demonstrated by the superiority of cued subjects at both time intervals. Noncued subjects were almost completely unable to produce NRC items at the $10-\mathrm{sec}$ interval, but with $30 \mathrm{sec}$ they were greatly improved, although still well below the performance of cued subjects. The added time had no effect on NRC-item retrieval for cued subjects.

The pattern of recall of RC items is important for the evaluation of the hypothesis being tested by the experiment. A two-factor analysis of variance on the transformed data yielded, again, two significant main effects and a significant interaction: Time, $F(1,60)=10.97$, $\mathrm{p}<.01$; Cueing, $\mathrm{F}(1,60)=5.70, \mathrm{p}<.05$; and Time by Cueing, $F(1,60)=7.17, p<.01$ (for all effects, $M_{\mathrm{e}}=$ .2). The tests for simple main effects revealed that the effect of Cueing was significant only for the $10-\mathrm{sec}$ level of the time variable and that the effect of Time was significant only for the cued condition. Although noncued subjects recalled approximately the same number of RC items at both time intervals, they were superior to cued subjects only at the 10-sec interval. Both groups were equivalent when $30 \mathrm{sec}$ were allowed. These data indicate that the RC-item pool is available to both cued and noncued learners and support the hypothesis that the amount of time allowed for retrieval is an important factor in the assessment of item differences between cued and noncued groups.

\section{DISCUSSION}

In general, the results of this experiment demonstrate that list items can be effective retrieval cues for categorized words acquired under conditions of random presentation. Two aspects of the experimental procedure can be identified which were most likely responsible for the observation of a cueing effect. First, following the suggestion of Allen (1969), a high degree of organization was allowed to develop by continuing acquisition over a number of trials. This should guarantee that list items had been encoded according to their category membership and that one item from a category might therefore be expected to cue retrieval of others. Although category clustering was not measured, inspection of the recall protocols indicated that the subjects were making extensive use of the taxonomic categories in organizing their output. Second, a 48-h retention interval was included to insure that a number of categories would be potentially available when cues were provided, but not accessible in free recall. The cueing procedure took advantage of the forgetting that had occurred and provided cues which were highly likely to be congruent with the organizational structure elaborated by the subject himself. It is not unexpected, then, that intralist cues were measurably effective under these conditions. However, if these can be viewed as the conditions under which experimenter-provided cues will be effective in general, why are these conditions more easily met with blocked presentation than with random presentation?

The characteristics of recall as a function of the method of presentation of categorized word lists have been examined by a number of investigators (e.g., Cofer, Bruce, \& Reicher, 1966; D'Agostino, 1969). With blocked presentation, output organization in the form of category clustering is typically very high on the first recall trial and measurable changes over trials are minimal. With random presentation, category clustering is observed to a lesser extent on the first trial of free recall and rapid increases occur over trials, reaching a level approaching that of a comparable blocked presentation group (Cofer, Bruce, \& Reicher, 1966; Hartley, 1974). To the extent that output organization reflects encoding strategies used by subjects, this evidence suggests that strategies used by blocked and random groups are somewhat different, at least in the early stages of learning, with a blocked group relying more heavily on the categorical structure of the list. Thus, subject- and experimenter-defined organizational structures are more congruent with blocked presentation than with random presentation, and the selection of an appropriate retrieval cue from the list is more certain for blocked groups, especially following a single study trial.

In addition, as noted previously, the tendency for more categories to be represented in the protocols of random presentation groups reduces the pool of possible categories which may be cued by an experimental manipulation. It is not clear why category recall should differ with blocked and random presentation. Perhaps with random presentation a more elaborate retrieval network develops, with items from a given category associated through contiguity of input with items from many other categories (Wallace, 1970). While these associations may interfere with the encoding of items by category membership, especially early in learning, they might persist and guarantee that at least some of the items from most categories are retrieved during free recall in later trials. Blocked groups would be expected to develop fewer of these alternate retrieval pathways and perhaps be more likely to forget some of the categories in a free-recall period.

The results of the item-type analyses can be interpreted in terms of the influence of cues on timedependent characteristics of retrieval strategies employed by cued and noncued subjects during an ex- 
tended memory search. When time is relatively limited, noncued subjects appear to direct their search toward the categories they have already recalled during Phase 1 , picking up a certain number of missed items from these categories. With additional time, little more can be gained from this strategy, but expansion of the search begins to reap benefits by allowing the searcher to access some previously forgotten categories. It would seem reasonable to predict that with sufficient time noncued subjects might be capable of producing most of the forgotten items.

Cued subjects, on the other hand, appear to use a strategy which is sequentially the reverse of that used by noncued subjects. When the experimenter provides an item from a nonrecalled category, the subject immediately directs his search to the forgotten category. With three words per category, $10 \mathrm{sec}$ was sufficient time for the subject to empty the available contents of the category once access was guaranteed. With added time, cued subjects showed little change with respect to recall of NRC items. However, cued subjects were quite capable of abandoning the no longer productive cue and engaging in a self-directed search strategy. The primary benefit of additional time was due to an increase in the number of $\mathrm{RC}$ items retrieved. With the longer interval of time, no differences were observed between cued and noncued groups in retrieval of RC items; the overall advantage for cued subjects was completely accounted for by retrieval of NRC items.

In summary, the results of this experiment suggest that, in addition to selecting appropriate cues and insuring that there is information available for cueing, care must be taken to minimize the time constraints imposed by a cueing procedure. This would seem to be particularly important when there are few forgotten chunks of information which might benefit from cues. Subjects apparently attempt to utilize a cue before engaging in a self-directed search. If the amount of time allowed is inadequate, a positive effect of cues might be obscured by the corresponding failure to produce the kinds of items generated during a self-directed search by a noncued comparison group.

\section{REFERENCES}

ALLEN, M. A. Cueing and retrieval in free recall. Joumal of Experimental Psychology, 1\%9, 81, 29-35.

Battig, W. F., \& Montague, W. E. Category norms for verbal items in 56 categories: A replication and extension of the Connecticut category norms. Joumal of Experimental Psychology Monograph, 1969, 80(No. 3, Part 2), 1-46.

Birnbaum, 1. M., \& Eichner, J. T. Study versus test trials and long-term retention in free-recall learning. Joumal of Verbal Learning and Verbal Behavior, 1971, 10, 516-521.

Borges, M. A., \& MANdLer, G. Effect of within-category spacing on free recall. Joumal of Experimental Psychology, 1972, 92, 207-214.

BousfiELD, W. A. The occurrence of clustering in the recall of randomly artanged associates. Joumal of General Psychology, 1953, 49, 229-240.

Cofer, C. N., Bruce, D. R., \& Reicher, G. M. Clustering in free recall as a function of certain methodological variations. Joumal of Experimental Psychology, 1966, 71, 858-866.

D'Agostino, P. R. The blocked-random effect in recall and recognition. Journal of Verbal Learning and Verbal Behavior. $1969,8,815-820$

Hartley, J. T. Study and test trials in free-reca!l learning (Doctoral dissertation, University of California, Irvine, 1973). Dissertation Abstracts International, 1974, 35, 542B (University Microfilms No. 74-13,839).

Hudson, R. L., \& Austin, J. B. Effect of context and category name on the recall of categorized word lists. Joumal of Experimental Psychology, 1970, 86, 43-47.

KIRK, R. E. Experimental design: Procedures for the behavioral sciences. Belmont: Brooks/Cole, 1968.

LEwIS, M. Q. Categorized lists and cued recall. Joumal of Experimental Psychology, 1971, 87, 129-131.

LEWIs, M. Q. Cue effectiveness in cued recall. Joumal of Experimental Psychology, 1974, 102, 737-739.

Luek, S. P., Mclaughlin, J. P., \& Cicala, G. A. Effects of blocking of input and blocking of retrieval cues on free recall learning. Journal of Experimental Psychology, 1971, 91, 159-161.

SLAmeCKA, N. J. An examination of trace storage in free recall. Joumal of Experimental Psychology, 1968, 76, 504-513.

SLAMECKA, N. J. The question of associative growth in the learning of categorized material. Joumal of Verbal Leaming and Verbal Behavior, 1972, 11, 324-332.

Tulvivg, E. Subjective organization in free recall of "unrelated" words. Psychological Review, 1962, 69, 344-354.

Tulving, E., \& Thомson, D. M. Encoding specificity and retrieval processes in episodic memory. Psychological Review, $1973,80,352.373$

Wallace. W. P. Consistency of emission order in free recall. Journal of Verbal Learning and Verbal Behavior, 1970, 9 58-68.

WooD, G. Retrieval cues and the accessibility of higher-order memory units in multitrial free recall. Joumal of Verbal Learning and Verbal Behavior, 1969, 8, 782-789.

(Received for publication October 6, 1975; revision accepted November $18,1975$. 